\title{
Surgical Treatment of Greater Saphenous Vein Thrombosis Involving the Saphenofemoral Junction: An Albanian Experience
}

Authors:

Disclosure:

Received:

Accepted:

Keywords:

Citation:
Sokol Xhepa, ${ }^{*}$ Gentian Vyshka ${ }^{2}$

1. Service of Vascular Surgery, University Hospital Centre "Mother Theresa",

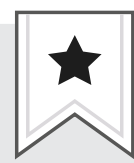

Tirana, Albania

2. Biomedical and Experimental Department, Faculty of Medicine, University of Medicine, Tirana, Albania

*Correspondence to gvyshka@yahoo.com

The authors have declared no conflicts of interest.

08.03.21

09.04 .21

Anticoagulants, pulmonary embolism, saphenofemoral junction, superficial vein thrombosis (SVT).

EMJ Int Cardiol. 2021;9[1]:49-50
Superficial vein thrombosis (SVT) is less wellstudied than deep vein thrombosis (DVT) because it has been considered less serious and is easily diagnosed following clinical symptomatology, and therefore requires mainly conservative treatment. ${ }^{1}$ The condition is common and is usually accompanied by clear inflammatory skin changes, and should be denoted as interchangeable with superficial vein thrombophlebitis. ${ }^{2}$

The saphenofemoral junction is an important anatomic marker when differentiating between SVT and DVT, the latter being a highly probable complication of the initial superficial thrombotic process. Authors diverge in the cut-off values of distance from the junction for the thrombotic process to be considered as a DVT with high probability of lethal consequences, such as a pulmonary embolism. Even in the absence of such a serious complication, DVT can still concur with SVT when thrombus presence is $1-3 \mathrm{~cm}$ from the junction. ${ }^{3-5}$

Authors still debate over the best treatment option for SVT. A diversity of methods and interventions are advised, including elastic stockings, anti-inflammatory drugs, anticoagulants, and/or surgery. ${ }^{6}$ Even surgeons are not unanimous when offering such a solution: ligation of the saphenofemoral junction (SFJ), stripping of the varicose veins, and resection of the greater saphenous vein (GSV). ${ }^{7,8}$ The recurrence of varicosities is in fact not the only adversity challenging almost all surgical options; pulmonary embolism is another major event that requires appropriate prevention peri- and intra-operatively, since an extension of the thrombus into the deep venous system is still possible. Apart from concerns over these adversities, authors still debate the best option for treatment of SVT, as well as the best technique, if any. ${ }^{9}$

From January 2012 to December 2016, 120 patients presented at the Service of Vascular Surgery, Tirana, Albania, with truncal varicose veins complicated with thrombosis within 0-5 $\mathrm{cm}$ from the SFJ. The mean age of patients was 58 years (range: $34-82$ years) with the majority of patients in their 50s. 82 patients were female (68\%) and 38 were male (32\%). All cases underwent a duplex scan pre-operatively, and the imaging data were associated with the intraoperative findings. 
In 24 patients (20\% of the cases), in whom the thrombus was $1 \mathrm{~cm}$ from the SFJ and with extension into the common femoral vein (CFV), a high ligation was performed after clamping the CFV above and below the junction. In the other group of patients ( $n=96 ; 80 \%$ of the cases), where the thrombus was $2-5 \mathrm{~cm}$ from the SFJ, the CFV was not clamped. The presence of the thrombus within $0-5 \mathrm{~cm}$ from the SFJ is likely to be an indication for surgical intervention, albeit other sources suggesting the efficacy of a 45day anticoagulation therapy. ${ }^{10}$

The disconnection of the GSV from the CFV will prevent the extension of the thrombus into the femoral and iliac veins, thus avoiding potentially life-threatening complications such as DVT and pulmonary embolism. What is more, the clamping of the CFV should always be performed when the thrombus has penetrated the later, when it is within $1 \mathrm{~cm}$ from the junction, and when the surgeon is not sure whether the junction itself is free. On the other hand, when sonography data suggest a thrombus distance of $2-5 \mathrm{~cm}$ from the femoral vein, there is enough space for the operating surgeon to ligate the saphenous vein without risking a thromboembolic event, and no need to clamp the CFV.

None of the patients suffered from a pulmonary embolism during the procedures. In the second subgroup (96 patients; operated without clamping the CFV) there was one case with DVT, with extension of the thrombotic process into the common femoral and external iliac veins. Another two patients from the same subgroup presented minor symptomatology of pulmonary embolism post-operatively. All three cases with minor complications were successfully treated with rivaroxaban and were discharged within 1 week following the operation. The other patients (117/120) were discharged 2-3 days following the surgery. No serious complications presented in either subgroup within a 3-month follow-up period.

Surgery of GSV involving SFJ is a safe and effective option, when comparing final outcomes with anticoagulation therapy alone; the occurrence of severe adverse complications when anticoagulating patients should also be kept in mind.1

\section{References}

1. Cosmi B. Management of superficial vein thrombosis. J Thromb Haemost. 2015;13(7):1175-83.

2. Kim $\mathrm{SH}$ et al. Isolated proximal greater saphenous vein thrombosis and the risk of propagation to deep vein thrombosis and pulmonary embolism. Vasc Health Risk Manag. 2018;14:129-35.

3. Ascer $\mathrm{E}$ et al. Preliminary results of a nonoperative approach to saphenofemoral junction thrombophlebitis. J Vasc Surg. 1995;22(5):616-21.

4. Lohr JM et al. Operative management of greater saphenous thrombophlebitis involving the saphenofemoral junction. Am J Surg. 1992;164(3):269-75.

5. Prandoni P et al. High vs. low doses of low-molecular-weight heparin for the treatment of superficial vein thrombosis of the legs: a doubleblind, randomized trial. J Thromb Haemost. 2005;3(6):1152-7.

6. Karathanos $\mathrm{C}$ et al. Patterns in the management of superficial vein thrombosis. Phlebology. 2017;32(3):207-13.

7. Mumme $A$ et al. High ligation of the saphenofemoral junction is necessary! Phlebologie. 2009;38(3):99-102.

8. Cappelli $\mathrm{M}$ et al. Ligation of the saphenofemoral junction tributaries as risk factor for groin recurrence. $\mathrm{J}$ Vasc Surg Venous Lymphat Disord. 2018;6(2):224-9.

9. Sullivan $\vee$ et al. Ligation versus anticoagulation: treatment of aboveknee superficial thrombophlebitis not involving the deep venous system. J Am Coll Surg. 2001;193(5):556-62.

10. Decousus $\mathrm{H}$ et al. Epidemiology, diagnosis, treatment and management of superficial-vein thrombosis of the legs. Best Pract Res Clin Haematol. 2012;25(3):275-84.

11. Sabanis $\mathrm{N}$ et al. Fondaparinuxassociated rectus sheath hematoma: skating on thin ice. Cureus. 2020;12(5):e7938. 